\title{
A Fuzzy Indoor Positioning System with ZigBee Wireless Sensors
}

\author{
Chih-Yung Chen ${ }^{1}$, Yu-Ju Chen ${ }^{2}$, Shen-Whan Chen ${ }^{3}$, Chi-Yen Shen ${ }^{4}$, Rey-Chue Hwang ${ }^{4}$ * \\ ${ }^{1}$ Department of Computer and Communication, Shu-Te University, Kaohsiung City, Taiwan \\ ${ }^{2}$ Department of Information Management, Cheng Shiu University, Kaohsiung City, Taiwan \\ ${ }^{3}$ Department of Communication Engineering, I-Shou University, Kaohsiung City, Taiwan \\ ${ }^{4}$ Department of Electrical Engineering, I-Shou University, Kaohsiung City, Taiwan
}

Email address:

mikechen@stu.edu.tw (Chih-Yung Chen), yjchen@csu.edu.tw (Yu-Ju Chen), jasonchen@isu.edu.tw (Shen-Whan Chen), cyshen@isu.edu.tw (Chi-Yen Shen ), rchwang@isu.edu.tw (Rey-Chue Hwang)

${ }^{*}$ Corresponding author

\section{To cite this article:}

Chih-Yung Chen, Yu-Ju Chen, Shen-Whan Chen, Chi-Yen Shen, Rey-Chue Hwang. A Fuzzy Indoor Positioning System with ZigBee Wireless Sensors. Journal of Electrical and Electronic Engineering. Vol. 4, No. 5, 2016, pp. 97-102. doi: 10.11648/j.jeee.20160405.12

Received: September 21, 2016; Accepted: October 14, 2016; Published: October 20, 2016

\begin{abstract}
This paper presents an indoor positioning system (IPS) by using fuzzy estimation technique. The research aims to design an IPS with high stability, high accuracy and high reliability. The received signal strengths (RSS) sensed by ZigBee wireless sensors were used to estimate the object's position. All studies were experimented at a 10x10 square meters' indoor field. In all experiments, 361 positions (features) were estimated. From the experimental results shown, the fuzzy positioning technique proposed has the high accurate estimation even RSS signals are unstable. It is also clearly found that the positioning accuracy could be greatly improved when more wireless sensors are used in IPS.
\end{abstract}

Keywords: Indoor Positioning System, Fuzzy, ZigBee Wireless Sensor

\section{Introduction}

In recent years, the positioning technique has been widely used for the location-based services (LBS), such as navigation, tourism, vehicle and military applications, etc. In order to make LBS more efficient and accurate, the wireless communication elements and techniques have been widely employed in developing the positioning system. Global Positioning System (GPS) is the most popular technique used in outdoor positioning service. It is the worldwide satellite-based radio navigation system. The system's satellites transmit the navigation messages to a GPS receiver which is used to determine the target's position. Generally, GPS uses triangulation method to find the target's position in accordance with the distances between GPS hand-held receiver and satellites [1-3]. Thus, it needs to obtain the distance information at least three or four satellites. However, GPS is a line-of-sight transmission system. The satellite signals are easily deteriorated at indoor environment due to the external interferences like obstacle, wall and electrical equipment, etc. Therefore, GPS is seldom used in the indoor positioning application.

The indoor positioning problem has attracted the researchers' interest recently. IPS has been employed into many applications, such as the rescue operation, patient monitoring, cargo management, public guiding system and so on. IPS is able to provide the location information about the persons or devices. Compare with the outdoor positioning system, IPS is a positioning system which needs much higher degree of accuracy to object's position.

Figure 1 shows the example about the concept of IPS application. The object's coordinate needs to be determined is $\left(x_{o}, y_{o}\right) . \mathrm{A}, \mathrm{B}$, and $\mathrm{C}$ are three wireless sensors and their corresponding coordinates are, $\left(x_{A}, y_{A}\right),\left(x_{B}, y_{B}\right)$ and $\left(x_{C}, y_{C}\right)$, respectively. $r_{A}, r_{B}$ and $r_{C}$ could be the distances or RSS signals between the object and sensors [4]. The object's position $\left(x_{o}, y_{o}\right)$ then can be estimated by using positioning algorithm in accordance with the information $\left(\left(x_{A}, y_{A}\right),\left(x_{B}, y_{B}\right)\right.$, $\left(x_{C}, y_{C}\right), r_{A}, r_{B}$ and $\left.r_{C}\right)$ of sensors $\mathrm{A}, \mathrm{B}$, and $\mathrm{C}$. 


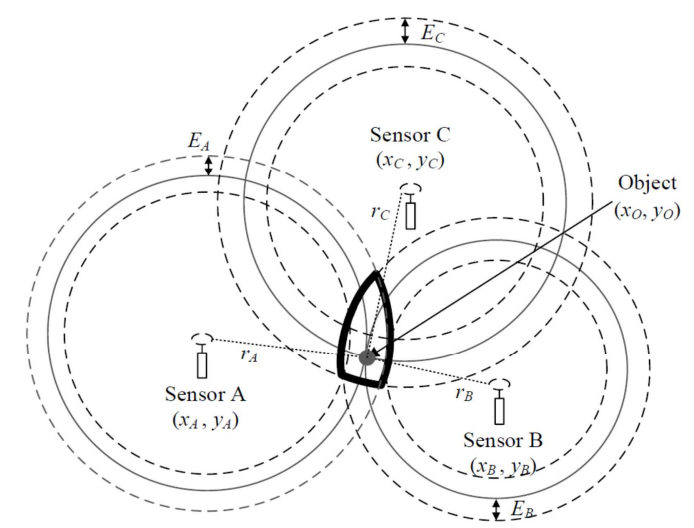

Figure 1. The relationship between unknown object and wireless sensors.

Basically, IPS consists of two important parts, i.e. the sensing infrastructure and the position estimation algorithm. The sensing infrastructure is mainly used to transmit or receive the sensing signal that can be used by positioning algorithm for estimating the object's position. In IPS system, the sensing infrastructure is composed of the wireless communication hardware and technology.

Nowadays, many wireless communication technologies such as wireless local area network (WLAN) [5-8], wireless sensor network (WSN) [9-10], radio frequency identification (RFID) [11-13], Bluetooth [14-15], ZigBee [16-17], etc. have been wildly used in developing IPS. However, due to the limitation of the function of sensing components, different IPSs have different advantages while they are used in the various positioning applications. Some IPSs even need to take the trade-off between the performance and the real condition of environment.

The positioning algorithm is about the software used for estimating the object's position. In the past decade, many positioning algorithms have been proposed. In which, three positioning algorithms, including triangulation, scene analysis and proximity, are well-known methods used in IPS [18-23]. Basically, the values of RSS sensed from the known reference nodes are usually taken to calculate the object's coordinate [24-26]. But, due to the influences of external factors such as the disturbances of hindrance, noise and the diffraction of electromagnetic wave, the proper positioning estimation method is still a challenging topic in the research of IPS application.

In this study, a fuzzy estimation algorithm is proposed for the indoor positioning application. Based on RSS signals sensed, the fuzzy estimator could accurately determine the object's position. The whole paper is organized as follows. In Section 2, the indoor positioning system with ZigBee sensing module is presented. Section 3 describes how the fuzzy estimation algorithm is used for the positioning calculation. Section 4 presents the relevant experiments and results of fuzzy IPS proposed. At last, a conclusion is given in Section 5.

\section{The Indoor Positioning System}

The sensing infrastructure in this research is a ZigBee module produced by Texas Instruments (TI) Corporation as shown in Figure 2 [4]. ZigBee is an IEEE 802.15.4-based specification for a suite of high-level communication protocols used to create personal area networks with small and low-power digital radios. It is intended to be simpler and less cost than other wireless personal area networks. This module includes an 8-bit CPU core which is an enhanced version of the industry standard 8051 core. The antenna is isotropic and its working frequency is $2.4 \mathrm{GHz}$. The RF chip is CC2430F 128 / CC2431.

The developed sensing module includes location dongle, blind node, and reference node. The location dongle can capture RSS between blind node and all reference nodes. The application programming interface (API) provided by TI allows user to obtain blind node's location through the RSS signals on ZigBee module. The RSS indicator (RSSI) is usually measured in $\mathrm{dBm}$ and has the typical values within interval $[-40 \mathrm{dBm},-95 \mathrm{dBm}]$. The industry standard always defines RSS value by 256 intervals. This research aims to design an accurate IPS based on ZigBee sensing module. This IPS is expected to have the better performance than current positioning methods.

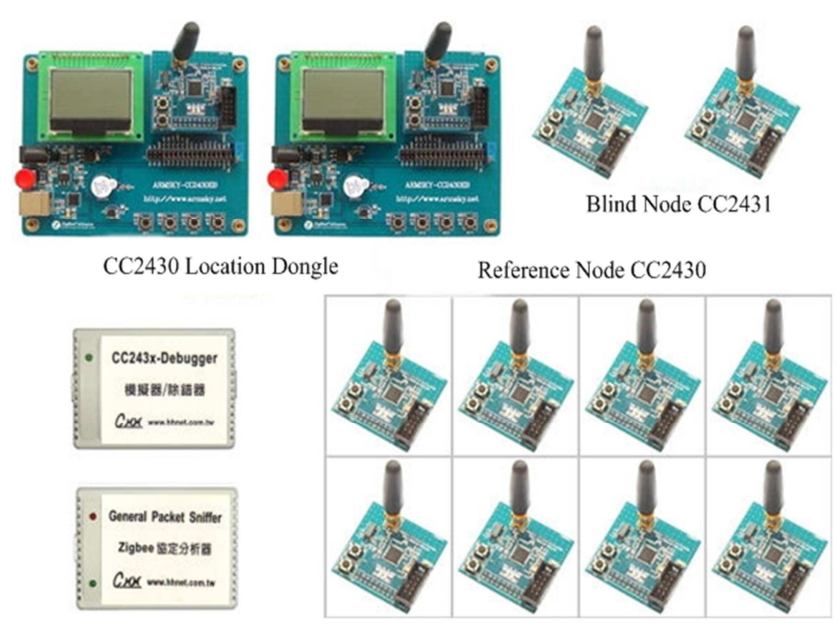

Figure 2. Zigbee sensing infrastructure.

\section{Fuzzy Positioning Algorithm}

Fuzzy theory was initialized by Professor Lotfi Zadeh in 1965. It has been applied into many fields ranging from control, signal processing, communications, and expert systems to business, medicine, etc [27-33]. It is a theory to formulate human knowledge in a systematic manner. Thus, the fuzzy system is a knowledge-based and rule-based system. The main knowledge base consists of many fuzzy IF-THEN rules which are created based on expert's experience.

Basically, a well- defined fuzzy system is composed of four parts, i.e. fuzzifier, fuzzy rule base, fuzzy inference engine and defuzzifier. Fuzzifer plays the role to map a real-valued information to a fuzzy set. Contrary, defuzzifer is a tool to map a fuzzy set to a crisp real value. The fuzzy rule base consists of a set of fuzzy IF-THEN rules. It is constructed based on the knowledge or experience of the 
expert. It plays the heart of the whole fuzzy system. The fuzzy inference engine is a fuzzy logic principle which can combine the fuzzy IF-THEN rules into a mapping from a fuzzy set in fuzzifier to a fuzzy set of defuzzifier.

Figure 3 shows the standard fuzzy logic system. The fuzzy system could provide a systematic procedure for transforming a knowledge base into a nonlinear mapping.

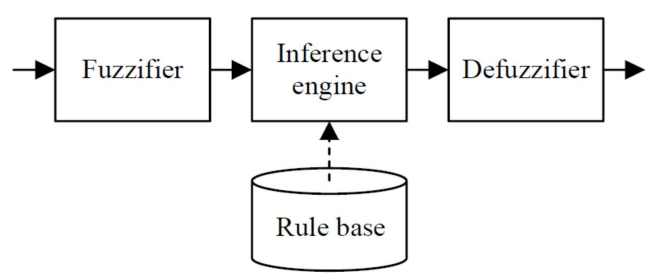

Figure 3. The standard fuzzy logic system.

In our study, the coordinate of object will be estimated by the fuzzy positioning algorithm proposed. At here, we use IPS with 3 ZigBee sensors to be an example for interpreting the whole fuzzy positioning algorithm. Let $\mathbf{C}$ be a set of class vectors, i.e. IPS database, which is given by

$$
\mathbf{C}=\left\{\begin{array}{l}
\left(\left(x_{1}, y_{1}\right),\left(R S S_{11}, R S S_{12}, R S S_{13}\right)\right), \\
\left(\left(x_{2}, y_{2}\right),\left(R S S_{21}, R S S_{22}, R S S_{23}\right)\right), \\
\ldots,\left(\left(x_{m}, y_{m}\right),\left(R S S_{m 1}, R S S_{m 2}, R S S_{m 3}\right)\right)
\end{array}\right\}
$$

$\left\{\left(x_{1}, y_{1}\right),\left(x_{2}, y_{2}\right), \ldots,\left(\left(x_{m}, y_{m}\right)\right\}\right.$ are the coordinates of reference positions and $\left\{\left(R S S_{11}, R S S_{12}, R S S_{13}\right), \ldots,\left(\left(R S S_{m 1}\right.\right.\right.$, $\left.\left.R S S_{m 2}, R S S_{m 3}\right)\right\}$ are RSS signals sensed on the reference nodes. Now, we assume RSS signal vector sensed at a blind node $\left(x_{b}\right.$, $\left.y_{b}\right)$ is $\left(R S S_{b 1}, R S S_{b 2}, R S S_{b 3}\right)$, then the fuzzy rule base can be constructed as the following $\mathrm{m}$ fuzzy IF-THEN rules.

$\mathrm{Ru}^{(1)}$ : IF $R S S_{b 1}$ is $R S S_{11}$ and $R S S_{b 2}$ is $R S S_{12}$ and $R S S_{b 3}$ is $R S S_{13}$, THEN $\left(x_{b}=x_{1}, y_{b}=y_{1}\right)$.

$\mathrm{Ru}^{(2)}$ : IF $R S S_{b 1}$ is $R S S_{21}$ and $R S S_{b 2}$ is $R S S_{22}$ and $R S S_{\underline{b} 3}$ is $R S S_{23}, \operatorname{THEN}\left(x_{b}=x_{2}, y_{b}=y_{2}\right)$.

$\mathrm{Ru}^{(\mathrm{m})}$ : IF $R S S_{b 1}$ is $R S S_{m 1}$ and $R S S_{b 2}$ is $R S S_{m 2}$ and $R S S_{b 3}$ is $R S S_{m 3}$, THEN $\left(x_{b}=x_{\mathrm{m}}, y_{b}=y_{\mathrm{m}}\right)$.

In this research, there are 361 points in the database, thus $m$ $=1,2, \ldots, 361$. In our fuzzy mechanism, the fuzzifier is Gaussian function which can be expressed by

$$
\begin{aligned}
& \mu\left(R S S_{m i}, \sigma\right) \\
& =\exp \left(-\frac{\left(R S S_{b i}-R S S_{m i}\right)^{T}\left(R S S_{b i}-R S S_{m i}\right)}{2 \sigma^{2}}\right), i=1,2,3 .
\end{aligned}
$$

where $\sigma$ is the smoothing parameter of Gaussian function.

The fuzzy inference engine is the combination of the product inference engine with algebraic product for $t$-norm operator. The final fuzzy relation inference value is computed as

$$
\mu\left(R S S_{m}, \sigma\right)=\mu\left(R S S_{m 1}, \sigma\right) * \mu\left(R S S_{m 2}, \sigma\right)^{*} \mu\left(R S S_{m 3}, \sigma\right)
$$

The center average defuzzifier is used to estimate the axes of object position.

$$
\begin{array}{r}
x_{b}=\frac{\sum_{m=1}^{361} x_{m} \mu\left(R S S_{m}, \sigma\right)}{\sum_{m=1}^{361} \mu\left(R S S_{m}, \sigma\right)} \\
y_{b}=\frac{\sum_{m=1}^{361} y_{m} \mu\left(R S S_{m}, \sigma\right)}{\sum_{m=1}^{361} \mu\left(R S S_{m}, \sigma\right)}
\end{array}
$$

\section{Experiments and Results}

In this research, a 10x10 square meter indoor field shown in Figure 4 is used for all experiments. In order to test the indoor positioning system developed, 3 to 8 sensors were experimented. The installations of 3 to 8 sensors are presented in Figure 5(a) to Figure 5(f).

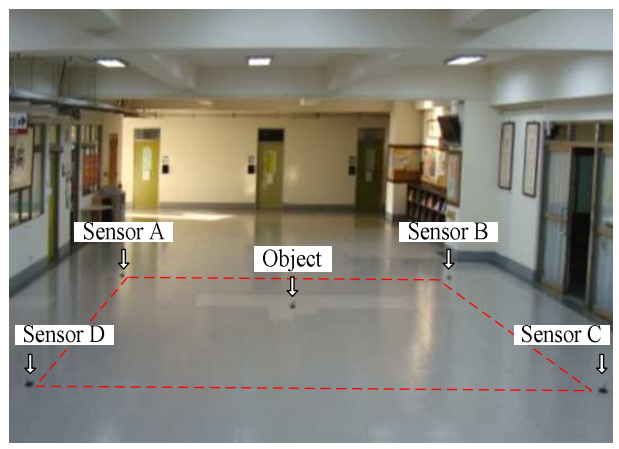

Figure. 4. The field of experimental environment.

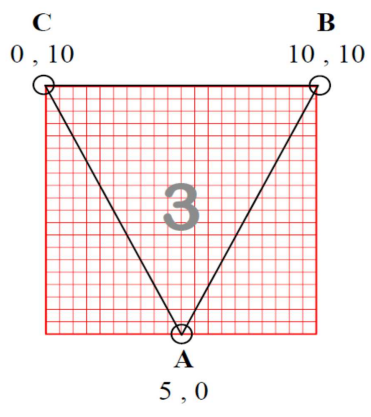

(a) 3 sensors

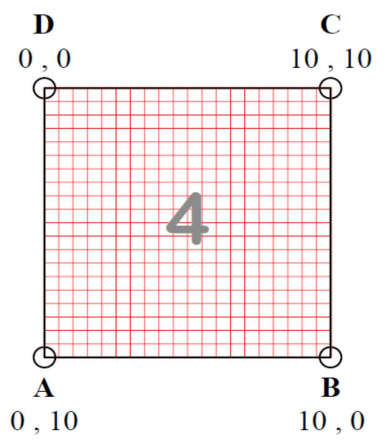

(b) 4 sensors 


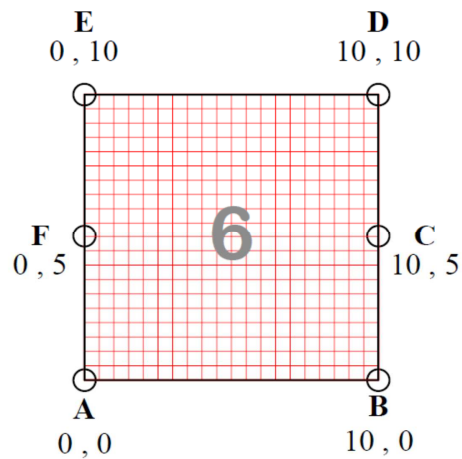

(c) 5 sensors

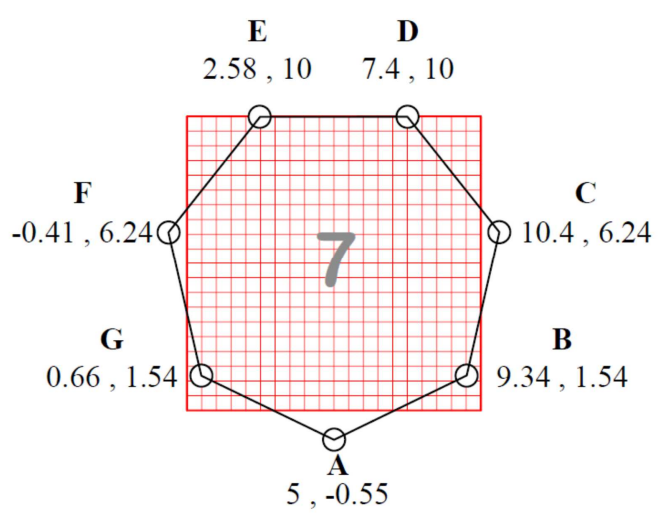

(d) 6 sensors

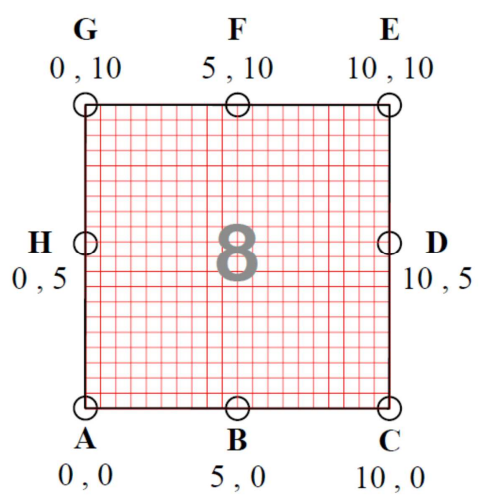

(e) 7 sensors

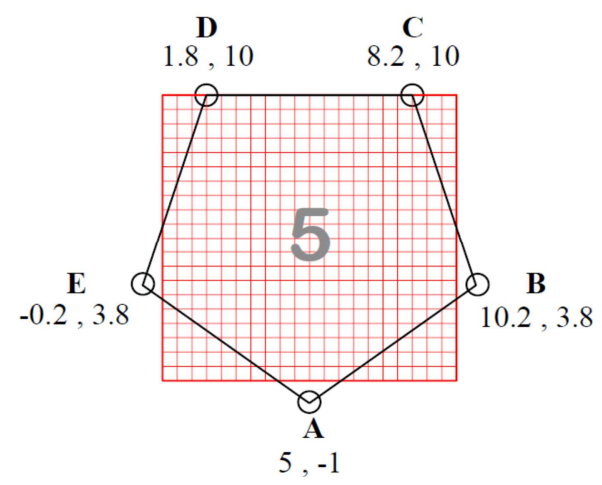

(f) 8 sensors

Figure 5. The installations of sensors on $10 \times 10$ square meters.
For each experiment, 361 positions (features) were measured twice at different time periods. First 361-point data are treated as the database which has the correct information about object's positions. Second 361-point data are used to test the accuracy of fuzzy positioning algorithm developed. The mean absolute error (MAE) is used as the estimated measurement for all experiments.

Table 1 lists the positioning errors for three different sensing experiments ( 3,4 and 5 sensors). Table 2 lists the positioning errors for another three different sensing experiments (6, 7 and 8 sensors). From the results shown, all fuzzy positioning systems with $\sigma=0.01$ have the best estimations. The best positioning errors could reach to $4.28615 \mathrm{~cm}$ and $4.4500656 \mathrm{~cm}$ when 7 and 8 sensors are used. The results also show that the positioning accuracy could be greatly improved when more wireless sensors are used.

Table 1. The positioning errors for 3 sensing experiments.

\begin{tabular}{llll}
\hline & 3 sensors & 4 sensors & 5 sensors \\
\cline { 2 - 4 }$\sigma$ & $\begin{array}{l}\text { Test MAE } \\
(\mathbf{c m})\end{array}$ & $\begin{array}{l}\text { Test MAE } \\
(\mathbf{c m})\end{array}$ & $\begin{array}{l}\text { Test MAE } \\
(\mathbf{c m})\end{array}$ \\
\hline 0.01 & 88.80103 & 38.176937 & 18.560503 \\
0.02 & 116.33662 & 78.10107 & 51.36161 \\
0.03 & 133.69957 & 102.70749 & 80.27438 \\
0.04 & 149.9761 & 119.284255 & 99.08961 \\
0.05 & 167.59096 & 135.38148 & 112.39689 \\
0.06 & 185.90794 & 153.91508 & 124.0873 \\
0.07 & 204.26816 & 174.38492 & 136.63463 \\
0.08 & 221.77913 & 195.50444 & 150.7597 \\
0.09 & 238.05418 & 215.79195 & 166.03413 \\
0.1 & 252.66867 & 234.2117 & 182.00522 \\
\hline
\end{tabular}

Table 2. The positioning errors for 3 sensing experiments.

\begin{tabular}{llll}
\hline & 6 sensors & 7 sensors & 8 sensors \\
\cline { 2 - 4 }$\sigma$ & $\begin{array}{l}\text { Test MAE } \\
(\mathbf{c m})\end{array}$ & $\begin{array}{l}\text { Test MAE } \\
(\mathbf{c m})\end{array}$ & $\begin{array}{l}\text { Test MAE } \\
(\mathbf{c m})\end{array}$ \\
\hline 0.01 & 15.955858 & 4.28615 & 4.4500656 \\
0.02 & 36.81886 & 17.01670 & 13.558619 \\
0.03 & 61.43962 & 41.54112 & 35.53836 \\
0.04 & 77.02817 & 59.88475 & 55.314682 \\
0.05 & 89.93386 & 73.09709 & 69.68032 \\
0.06 & 102.85562 & 85.00864 & 81.859024 \\
0.07 & 117.22324 & 97.08852 & 93.59841 \\
0.08 & 133.8382 & 109.83302 & 106.0178 \\
0.09 & 152.01962 & 123.767494 & 119.75758 \\
0.1 & 170.66835 & 138.82343 & 134.70271 \\
\hline
\end{tabular}

\section{Conclusion}

In this research, an IPS module with Zigbee sensors and fuzzy estimation technique is developed. The whole wireless sensing infrastructure is constructed by ZigBee sensors. The received signal strengths sensed by ZigBee sensors were used to estimate the object's position. From the experimental results shown, the high accuracy of positioning error could be performed by the IPS system developed. That means the positioning system we developed indeed has the potential in real application. The low cost indoor positioning system could be constructed due to the Zigbee sensors. However, in our research, all experiments were implemented under the 
environment has no serious problem about the effects of interference, diffraction or reflection. Thus, the real condition of unstable environment is still a challenge topic and it will be continuous in our future studies.

\section{Acknowledgements}

This research was supported by the Ministry of Science and Technology, Taiwan, under Contracts No. MOST 105-2221-E-366-005, No. MOST 105-2632-E-366-001 and No. MOST-105-2221-E-214-041.

\section{References}

[1] E. Kaplan and C. Hegarty, Understanding GPS: Principles and Applicatons, 2nd ed. Norwood, MA: Artech House, 2006.

[2] C. C. Lin, M. J. Chiu, C. C. Hsiao, R. G. Lee, and Y. S. Tsai, "Wireless health care service system for elderly with dementia," IEEE Trans. Inf. Technol. Biomed., pp. 696-704, 2006.

[3] M. Leblang, S. J. Dunham, and F. Pappalardi, "HMS scott ring laser gyro navigator integration," Proceedings of OCEANS 2003, vol. 1, pp. 538-543, 2003.

[4] J. P. Yang, The Applications of Artificial Intelligence Technique in the Signal Characteristics' Analysis, Ph.D. Thesis, I-Shou University, 2012.

[5] A. Kotanen, M. Hannikainen, H. Leppakoski, T. D. Hamalainen, "Positioning with IEEE 802.11b wireless LAN," In the Proceedings of 14th IEEE Proceedings on Personal, Indoor and Mobile Radio Communications, Beijing, China, pp. 2218-2222, 2003.

[6] Y. B. Xu, M. Zhou, L. Ma, "Hybrid FCM/ANN indoor location method in WLAN environment," In the Proceedings of IEEE Youth Conference on Information, Computing and Telecommunications, Beijing, China, pp. 475-478, 2009.

[7] V. Honkavirta, T. Perala, S. Ali-Loytty, R. Piche, "A comparative survey of WLAN location fingerprinting methods," In the Proceedings of 6th Workshop on Positioning, Navigation and Communication (WPNC'09), Hannover, Germany, pp. 243-251, 2009.

[8] M. Y. Umair, K. V. Ramana, D. K. Yang, "An enhanced K-Nearest Neighbor algorithm for indoor positioning systems in a WLAN," 2014 IEEE Computers, Communications and Its Applications, pp. 19-23, January 20, 2014.

[9] K. F. S. Wong, I. W. Tsang, V. Cheung, S. H. G. Chan, J. T. Kwok, "Position estimation for wireless sensor networks," In the Proceedings of IEEE Global Telecommunications Conference, MO, USA, pp. 2772-2776, 2005.

[10] S. Aomumpai, K. Kondee, C. Prommak, K. Kaemarungsi, "Optimal placement of reference nodes for wireless indoor positioning systems," 11th International Conference on Electrical Engineering, Electronics, Computer, Telecommunications and Information Technology. Paper no. 6839894, 2014.

[11] P. Bahl V. N. Padmanabhan, "RADAR: An in-building RF-based user location and tracking system," In the Proceedings of INFOCOM 2000, Nineteenth Annual Joint Conference of the IEEE Computer and Communications Societies, Tel Aviv, Israel, pp. 775-784, 2000.
[12] H. D. Chon, S. Jun, H. Jung, S. W. An, "Using RFID for accurate positioning," Journal of Global Positioning Systems, vol. 3, pp. 32-39. 2004.

[13] H. L. Ding, W. W. Y. Ng, P. P. K. Chan, D. L. Wu, X. L. Chen, D S. Yeung, "RFID indoor positioning using RBFNN with L-GEM," In the Proceedings of IEEE 2010 International Conference on Machine Learning and Cybernetics, Qingdao, China, pp. 1147-1152, 2010.

[14] A. K. M. M. Hossain, W. S. Soh, "A comprehensive study of Bluetooth signal parameters for localization," In the Proceedings of 18th Annual IEEE International Symposium on Personal, Indoor and Mobile Radio Communications (PIMRC'07), Athens, Greece, pp. 1-5, 2007.

[15] F. Subhan, H. Hasbullah, A. Rozyyev, S. T. Bakhsh, "Indoor positioning in Bluetooth networks using fingerprinting and lateration approach," In the Proceedings of 2011 International Conference on Information Science and Applications (ICISA), Jeju Island, Korea, pp. 1-9, 2001.

[16] W. P. Chen, X. F. Meng, “A cooperative localization scheme for Zigbee-based wireless sensor networks," In the Proceedings of 14th IEEE International Conference on Networks, Singapore, pp. 1-5, 2006.

[17] G. Goncalo, S. Helena, "Indoor location system using ZigBee technology," In the Proceedings of Third International Conference on Sensor Technologies and Applications, Athens/Glyfada, Greece, pp. 152-157, 2009.

[18] B. Kim, W. Bong, Y. C. Kim, "Indoor localization for Wi-Fi devices by cross-monitoring AP and weighted triangulation," In the Proceedings of IEEE Consumer Communications and Networking Conference (CCNC), NV, U.S.A., pp. 933-936, 2011.

[19] Y. Mo, Z. Z. Zhang, Y. Lu, G. Agha, "A novel technique for human traffic based radio map updating in Wi-Fi indoor positioning systems," KSII Transactions on Internet and Information Systems, vol. 9, no. 5, pp. 1881-1903, 2015.

[20] X. F. Jiang, C. J. Mike Liang, K. F. Chen, B. Zhang, J. Hsu, J. Liu, B. Cao, F. Zhao, "Design and evaluation of a wireless magnetic-based proximity detection platform for indoor applications," In the Proceedings of 11th ACM/IEEE International Conference on Information Processing in Sensor Networks (IPSN/SPOTS), Beijing, China, pp. 221-231, 2012.

[21] J. Hightower, G. Borriello, "Location sensing techniques," Technical Report UW CSE 2001-07-30, Department of Computer Science and Engineering, University of Washington, 2001.

[22] K. Kaemarungsi, P. Krishnamurthy, "Properties of indoor received signal strength for WLAN location fingerprinting," In the Proceedings of 1st Annual International Conference on Mobile and Ubiquitous Systems: Networking and Services (MobiQuitous '04), MA, USA, pp. 14-23, 2004.

[23] D. Focken, R. Stiefelhagen, "Towards vision-based 3-D people tracking in a smart room," In the Proceedings of 4th IEEE Intl Conference on Multimodal Interfaces, PA, USA, pp. 400-405, 2002.

[24] S. Merat, W. Almuhtadi, "Wireless network channel quality estimation inside reactor building using RSSI measurement of wireless sensor network," In the Proceedings of Canadian Conference on Electrical and Computer Engineering, Calgary, AB, Canada, pp. 339-341, 2009. 
[25] H. C. Chen, Y. J. Chen, C. Y. Chen, S. M. T. Wang, J. P. Yang, R. C. Hwang, "A new indoor positioning technique based on neural network," Advanced Science Letters, vol. 19, no. 7, pp. 2029-2033, 2013.

[26] C. Y. Chen, Y. J. Chen, Y. C. Weng, S. W. Chen, R. C. Hwang, "The sectored antenna array indoor positioning system with neural networks", Automation, Control and Intelligent Systems, vol. 4, no. 2, pp. 21-27, 2016.

[27] L. X. Wang, A Course in Fuzzy Systems and Control, Prentice-Hall, Englewood Cliffs, NJ, 1997.

[28] H. X. Li and H. B. Gatland, "Conventional fuzzy control and its enhancement", IEEE Trans. on Systems, Man, Cybern., Part B, vol. 26, No. 5, pp. 791-797, 1996.

[29] Y. Yang, C. Zhou, J. Ren, "Model reference adaptive robust fuzzy control for ship steering autopilot with uncertain non-linear systems", Applied Soft Computing, vol. 3 No. 4, pp. 305-316, 2003.
[30] D. Adrian, Allyson, "Fuzzy logic's diffusion in the study of business, the social sciences, philosophy, and medicine", 2000 Annual Conference of the North American Fuzzy Information Processing Society - NAFIPS, pp. 178-182, 2000.

[31] A. Corona Nakamura, R. Ruelas, D. Andina, B. Ojeda-Magaña, "A review of benefits of neuro-fuzzy systems applied in medicine", The 9th World Multi-Conference on Systemics, Cybernetics and Informatics, Proceedings, vol. 1, pp. 344-349, 2005.

[32] T. Margarita, "A distributed adaptive neuro-fuzzy network for chaotic time series prediction", Cybernetics and Information Technologies, vol. 15, No. 1, pp. 24-33, 2015.

[33] M. Leandro, G. Fernando, B. Rosangela, "Stock market volatility prediction using possibilistic fuzzy modeling", 2015 Latin-America Congress on Computational Intelligence, LA-CCI 2015, March 17, 2016, 2015. 